\title{
ATUAÇÃO FONOAUDIOLÓGICA NA SÍNDROME DA APNÉIA E HIPOPNÉIA OBSTRUTIVA DO SONO: RELATO DE CASO
}

\author{
Speech therapy in the obstructive sleep \\ apnea-hypopnea syndrome: case report
}

\author{
Letícia Maria de Paula Silva (1), Flávia Talini dos Santos Aureliano (2), Andréa Rodrigues Motta (3)
}

\begin{abstract}
RESUMO
Objetivo: verificar a contribuição da Fonoaudiologia no tratamento de uma paciente com a síndrome da apnéia e hipopnéia obstrutiva do sono. Métodos: relato de caso de um paciente do sexo feminino, de sessenta anos de idade, com a síndrome da apnéia e hipopnéia do sono, de grau grave, que se submeteu ao atendimento fonoaudiológico para melhora do quadro de apnéia/hipopnéia, ronco e cansaço diurno. Foram realizadas avaliação clínica de motricidade orofacial e polissonografia antes e após a fonoterapia. Com base na avaliação clínica foi elaborado um plano terapêutico que buscou propiciar à paciente relaxamento cervical e da musculatura supra-hióidea, melhora na aeração nasal, adequação do posicionamento e força de língua, fortalecimento dos músculos do palato mole e sua mobilidade, aumento de força da musculatura mastigatória, treino da mastigação bilateral alternada e abaixamento do osso hióide. Resultados: após 12 sessões de terapia fonoaudiológica, com duração de 40 minutos cada, pôde-se observar diminuição da tensão cervical, relaxamento da musculatura supra-hióidea, adequação do posicionamento do osso hióide, língua normotensa com dorso rebaixado, palato mole com mobilidade normal e mastigação adequada. A paciente relatou importante melhora no cansaço diurno. O resultado da segunda polissonografia indicou diminuição de 44 para 3 eventos por hora de apnéia e hipopnéia durante o sono, tendo o quadro passado do nível grave a um índice de distúrbio respiratório baixo não mais caracterizando doença apnéica do sono. Conclusão: a fonoterapia foi eficaz para o tratamento deste caso de síndrome da apnéia e hipopnéia do sono.
\end{abstract}

DESCRITORES: Síndromes da Apnéia do Sono; Fonoterapia; Polissonografia

\section{INTRODUÇÃO}

A síndrome da apnéia e hipopnéia obstrutiva do sono (SAHOS) é caracterizada por pausas respiratórias durante o sono, devido a múltiplos colapsos e/ou estreitamentos da via aérea superior, ocasionando queda na saturação do oxigênio sanguíneo e fragmentação do sono. Na apnéia obstrutiva ocorre uma parada total do fluxo aéreo por um

(1) Fonoaudióloga; Impulso Formação Diária e Centro de Estimulação Especial Brincar; Especialização em Motricidade Orofacial pelo CEFAC - Saúde e Educação de Belo Horizonte - MG.

(2) Fonoaudióloga; Núcleo de Saúde do Eldorado; Grupo de Apoio à Pesquisa Odontológica; Especialização em Motricidade Orofacial pelo CEFAC - Saúde e Educação de Belo Horizonte - MG.

(3) Fonoaudióloga; Professora Assistente do Departamento de Fonoaudiologia da Universidade Federal de Minas Gerais; Mestre em Fonoaudiologia pela Pontifícia Universidade Católica de São Paulo. período maior ou igual a 10 segundos, enquanto que na hipopnéia o fluxo aéreo encontra-se somente diminuído ${ }^{1}$.

Durante o sono os músculos da faringe e da língua tendem a se relaxar, assim como toda musculatura do corpo. Na SAHOS esse relaxamento gera uma obstrução da via aérea superior, dificultando o fluxo aéreo e causando ruídos (ronco) e pausas respiratórias. Ocorre baixa oxigenação pulmonar e cerebral, o que leva o sistema nervoso central a agir em busca da abertura das vias aéreas superiores. Nesse momento o indivíduo tem um forte ronco e um microdespertar, que apesar de inconsciente, é suficiente para fragmentar o sono ${ }^{2}$.

$\mathrm{Na}$ população geral, a prevalência do ronco é maior no sexo masculino; estima-se que $27 \%$ dos homens e $18 \%$ das mulheres ronquem ${ }^{3}$. Na faixa etária acima de 40 anos nota-se aumento da prevalência do ronco em ambos os sexos, $24 \%$ e $36 \%$, respectivamente em homens e mulheres ${ }^{4}$, 
sendo que as mulheres apresentam mais pausas respiratórias durante o sono que os homens, respectivamente, $16 \%$ e $11 \%$. A queixa de sonolência diurna praticamente se equipara entre os sexos masculino $30 \%$ e feminino $31 \%{ }^{3}$. Acredita-se que o ronco aumente progressivamente à medida que se aumenta a idade, sendo estas variáveis mais associadas no sexo masculino ${ }^{5}$.

Outros sintomas relacionados com a SAHOS são: hipertensão arterial, fragmentação do sono, refluxo gastroesofágico, insônia, arritmias cardíacas, enurese noturna, cefaléia matinal, queda no rendimento intelectual, sintomas depressivos, impotência sexual e até alterações da personalidade ${ }^{1-6}$.

São considerados fatores predisponentes para a ocorrência da SAHOS obesidade, sexo masculino, anormalidades craniofaciais, hipertrofia de tonsilas, obstrução nasal, anormalidades endócrinas e história familiar ${ }^{1}$. Considera-se que a SAHOS seja um fator de risco, independente, para hipertensão arterial e conseqüente morbidade cardiovascular na população geral ${ }^{7}$ e morte ${ }^{8}$.

O diagnóstico da SAHOS é realizado por meio da avaliação clínica e confirmado pelo estudo polissonográfico, que consiste na monitorização do sono durante uma noite permitindo a avaliação de vários parâmetros fisiológicos básicos como eletroencefalograma, eletro-oculograma, eletromiograma, fluxo aéreo, esforço respiratório, eletrocardiograma e oximetria ${ }^{1-9}$. Além disso, a polissonografia é importante para controle da doença, determinando a eficácia do tratamento realizado ${ }^{10}$. O estudo polissonográfico permite classificar a SAHOS de acordo com o número de paradas respiratórias, ou seja, o índice de apnéia e hipopnéia (IAH), apresentadas no intervalo de 60 minutos: leve (5 a 15 eventos), moderada (15 a 30 eventos) e grave (mais de 30 eventos) ${ }^{1}$. Estima-se que em média $75 \%$ dos pacientes que realizam polissonografia têm diagnóstico de SAHOS ${ }^{11}$.

O tratamento clínico da SAHOS pode ser dividido em medidas comportamentais como redução de peso, evitar uso de álcool e sedativos, alterar a posição do corpo durante o sono evitando decúbito dorsal, entre outras; uso de aparelhos intra-orais que reposicionam a língua e a mandíbula anteriormente evitando o colapso entre a base da língua e as paredes da faringe; uso de CPAP (Continuous Positive Airway Pressure), aparelho que mantém a pressão positiva contínua na faringe, evitando seu colapso ${ }^{12}$.

O tratamento cirúrgico na SAHOS compreende procedimentos que, direta ou indiretamente, buscam aumentar o tamanho das vias aéreas superio- res, pela modificação dos tecidos moles da faringe (amígdalas palatinas e linguais, palato mole, base da língua e epiglote) ou da modificação do esqueleto craniofacial (maxila, mandíbula, hióide) ${ }^{13}$. O tratamento cirúrgico tem prognóstico reservado para pacientes que apresentam grau moderado ou grave de apnéia obstrutiva do sono, índice de massa corporal elevado e retrognatia ${ }^{4}$.

Uma nova opção de tratamento para a SAHOS é a terapia fonoaudiológica que vem demonstrando resultados satisfatórios ${ }^{14}$, melhorando significativamente os sintomas iniciais e proporcionando uma qualidade de vida melhor num curto espaço de tempo. Em pacientes portadores de SAHOS, nota-se uma melhora de $40 \%$ a $50 \%$ no número de paradas respiratórias ${ }^{14}$, bem como uma diminuição considerável do ronco e da sonolência diurna após tratamento fonoaudiológico. O mesmo consiste em adequar aspectos anátomo-morfológicos e anátomo-funcionais dos órgãos fonoarticulatórios, que nessa doença, apresentam-se com tensão diminuída (flácidos) ${ }^{14}$. Observa-se relação entre respiração oral ou oronasal e apnéia do sono ${ }^{15}$, visto que uma respiração inadequada pode gerar fraqueza da musculatura orofaríngea ${ }^{16}$; nestes casos, o tratamento fonoaudiológico miofuncional vem auxiliar no tratamento da síndrome da apnéia e hipopnéia obstrutiva do sono ${ }^{15}$.

O presente estudo de caso teve como objetivo verificar a contribuição da Fonoaudiologia no tratamento de uma paciente com síndrome da apnéia e hipopnéia obstrutiva do sono de grau grave.

\section{MÉTODOS}

Este estudo foi baseado no relato de caso de um paciente do sexo feminino de sessenta anos, com diagnóstico otorrinolaringológico de SAHOS de grau grave, após ter sido submetido ao exame de polissonografia. Foram utilizados dados de avaliações, exames e tratamentos realizados por uma equipe interdisciplinar composta por otorrinolaringologista, ortodontista, ortopedista e fonoaudiólogo.

A paciente apresentava história pregressa de ronco há aproximadamente onze anos, com queixa principal de sonolência e cansaço diurno, apresentando adequado índice de massa corporal $(I M C=23,3)$. A paciente procurou tratamento por insistência dos familiares devido ao ronco de forte intensidade. Encontrava-se em acompanhamento otorrinolaringológico há quatro anos. A primeira polissonografia foi realizada em agosto de 2005 e como proposta terapêutica indicou-se o uso do CPAP (Contínuos Positive Airway Pressure) Nasal, 
porém houve grande dificuldade de adaptação, o que resultou em abandono do tratamento.

A paciente foi submetida à avaliação clínica fonoaudiológica em maio de 2006, tendo realizado também avaliação ortodôntica, ortopédica e exame complementar de fibronasolaringoscopia, no intuito de observar se havia alterações nas áreas acima pesquisadas que viessem a interferir no tratamento fonoaudiológico. Na avaliação ortodôntica observou-se que a paciente apresentava má oclusão de Classe II divisão I, com overjet e overbite ligeiramente aumentados e protrusão bimaxilar; tipo mesofacial com perfil bastante convexo sugerindo um corpo mandibular menor que o normal. O profissional indicou tratamento fonoaudiológico e otorrinolaringológico para melhora do quadro. $\mathrm{Na}$ avaliação ortopédica verificou-se que a paciente apresentava retificação da lordose fisiológica cervical, ao raio-x, não apresentando, entretanto, alterações clínicas e queixas importantes do aparelho músculo-esquelético.

Ao exame de fibronasolaringoscopia observouse: vestíbulos nasais e mucosa nasal sem alterações; conchas inferiores hipertróficas; base de língua, valécula, epiglote e seios piriformes sem alterações. Verificou-se ainda, desvio de septo nasal em área II e III para a esquerda tipo esporão, impactante em corneto inferior; colabamento láterolateral leve em região retropalatal e ausente retroglossal à Manobra de Müller.

A terapia fonoaudiológica compreendeu 12 sessões, tempo satisfatório para se observar evolução no padrão muscular, com atendimentos semanais de 40 minutos cada. Ao final do tratamento foi refeita a polissonografia, buscando quantificar objetivamente a melhora proporcionada pelo tratamento fonoaudiológico e, também a avaliação miofuncional orofacial para observar mudanças na tensão dos músculos trabalhados.

Este relato de caso foi aprovado pelo Comitê de Ética em Pesquisa do CEFAC - Saúde e Educação sob número 34/06, tendo sido considerado como sem risco e com necessidade do termo de consentimento livre e esclarecido.

Por se tratar de um estudo de caso, os dados foram analisados comparando-se, qualitativamente os dados fonoaudiológicos antes e após a intervenção, além de se ter observado as mudanças na polissonografia.

\section{RESULTADOS}

Em junho de 2006, quando realizada a anamnese, a paciente trouxe o resultado da polissonografia que havia sido realizada em agosto de 2005. Apesar de transcorridos 10 meses da realização do exame não foi possível a solicitação de nova avaliação ao início do tratamento fonoaudiológico por falta de cobertura do convênio médico. Entretanto, a paciente continuava a apresentar queixas de ronco, sonolência e cansaço diurno exatamente na mesma intensidade.

O primeiro exame de polissonografia evidenciou uma fragmentação do sono de intensidade severa (44 microacordares por hora), em decorrência especialmente das apnéias de caráter obstrutivo, independentes da posição corporal. Verificou-se ainda saturação média de oxigênio de $93 \%$, chegando a uma mínima de $83 \%$.

$\mathrm{Na}$ avaliação fonoaudiológica constatou-se postura inadequada de cabeça (inclinada para frente) e tensão cervical; bochechas assimétricas com maior tensão à esquerda; rigidez de musculatura supra-hióidea; posição elevada de osso hióide; masseter com discreta força e velocidade maior de contração à esquerda; temporal com discreta força e velocidade de contração maior à direita; língua com tensão diminuída em parte anterior e média, e dorso elevado e hipertenso; palato mole com mobilidade reduzida e mastigação unilateral à esquerda.

Após avaliação fonoaudiológica a paciente foi encaminhada à Ortodontia e Ortopedia. Cabe ressaltar que o convênio médico da paciente apresentava cobertura para fisioterapia apenas decorrente de acidentes, só tendo sido possível, portanto, solicitar avaliação ortopédica.

O tratamento fonoaudiológico foi realizado semanalmente durante três meses, tendo como um dos objetivos proporcionar à paciente um sono mais tranqüilo, reparador e com diminuição do número de apnéias, melhorando assim sua qualidade de vida.

Diante dos achados da avaliação fonoaudiológica foi elaborado um plano terapêutico que buscava propiciar à paciente relaxamento cervical e da musculatura supra-hióidea, melhora na aeração nasal, adequação do posicionamento e força de língua, fortalecimento dos músculos do palato mole e sua mobilidade, aumento de força da musculatura mastigatória, treino da mastigação bilateral alternada e abaixamento do osso hióide.

No início do tratamento foram utilizados alongamentos em região cervical e massoterapia em 
região supra-hióidea para diminuição da tensão e, massagens descendentes na região laríngea para abaixamento do osso hióide. Limpeza diária da cavidade nasal com soro fisiológico e massagens rotatórias na asa do nariz foram orientadas objetivando-se melhora da aeração nasal.

A partir da terceira sessão foram introduzidos exercícios isocinéticos e isométricos para musculatura da língua, buscando-se um posicionamento mais anteriorizado. Exercícios isocinéticos e isométricos foram empregados também nos músculos masseter, temporal e bucinador. Na quarta sessão os exercícios foram estendidos ao fechamento velofaríngeo, visando fortalecimento e mobilidade dos músculos do palato mole.

$\mathrm{Na}$ oitava sessão a paciente foi orientada sobre a mastigação bilateral alternada e deu-se inicio ao treino funcional com alimento, visto que os músculos já se encontravam com força adequada.

A partir da oitava sessão a paciente relatou melhora no cansaço e sonolência diurna, voltando a realizar caminhadas e atividades domésticas sem sentir fadiga, tendo seus familiares notado diminuição na intensidade do ronco.

Ao final das doze sessões propostas a avaliação miofuncional orofacial e a polissonografia foram refeitas. Na reavaliação clínica pôde-se observar diminuição da tensão cervical, relaxamento da musculatura supra-hióidea, adequação do posicionamento do osso hióide, língua normotensa com dorso rebaixado, palato mole com mobilidade normal e melhora da mastigação.

O exame polissonográfico teve resultado compatível com roncos e despertares moderados e índice de distúrbio respiratório baixo não caracterizando doença apnéica do sono. O índice de distúrbio respiratório (índice de apnéia e hipopnéia) foi de três eventos por hora com saturação média de $\mathrm{O} 2$ de $99 \%$, chegando à mínima de $92 \%$.

A paciente apresentava fatores agravantes que haviam determinado um prognóstico reservado para o caso. Foram observados no exame de fibronasolaringoscopia desvio de septo nasal e conchas inferiores hipertróficas, sendo, porém, a aeração nasal adequada. Além disso, apresentava uma má oclusão do tipo classe II divisão I, que gera diminuição do espaço intra-oral. Outro fator de grande relevância era a gravidade de sua doença, visto que não existem relatos na literatura com melhora significativa em pacientes com SAHOS de grau grave. Dessa forma, considerou-se os resultados da intervenção fonoaudiológica surpreendentes.

\section{DISCUSSÃO}

Dentre os fatores predisponentes para a SAHOS descritos na literatura ${ }^{1}$, verificou-se na paciente em estudo somente obstrução nasal e possível história familiar, visto que sua mãe apresentava ronco, mas não foi submetida a qualquer avaliação para diagnóstico de SAHOS. A paciente apresentava peso adequado para sua altura, respectivamente, $68 \mathrm{Kg} \mathrm{e}$ $1,71 \mathrm{~m}$, constatando-se diminuição de $2 \mathrm{Kg}$ durante o período de estudo, sem grande impacto no seu índice de massa corporal (IMC=22,6).

Estudos indicam aumento da prevalência dos sintomas da SAHOS acima de 40 anos ${ }^{4}$, sendo coerente com a história pregressa da paciente que começou a apresentar os primeiros sintomas aos 50 anos de idade.

Diante do resultado da polissonografia a paciente em estudo tentou adaptação do CPAP por uma semana, mas queixou-se da dificuldade para dormir com o aparelho devido ao incômodo e ruído que o mesmo provoca, abandonando, então, o seu uso. Há relatos de inúmeros casos de dificuldade de adesão ao CPAP devido à claustrofobia, obstrução, irritação e aumento de resistência de vias aéreas superiores, desconforto ou irritação facial pela máscara e pressão do fluxo aéreo ${ }^{17}$. Apesar de ser o tratamento clínico mais utilizado e de ser terapeuticamente efetivo, a adesão ao CPAP é insatisfatória ${ }^{2}$. O tratamento cirúrgico já foi indicado para a paciente, mas a mesma se recusou a realizá-lo. Atualmente sabe-se que o tratamento cirúrgico em casos moderados ou graves, como o do referido caso, tem prognóstico reservado com chances de recidiva ${ }^{4}$.

Durante o tratamento foram utilizados alongamentos em região cervical, visando conscientização e percepção postural, de acordo com o descrito na literatura ${ }^{18}$, além do relaxamento muscular. Foi indicada também limpeza diária da cavidade nasal com soro fisiológico para desobstrução e lavagem nasal ${ }^{18}$.

Empregou-se exercícios isométricos e isocinéticos para fortalecimento e coordenação dos músculos da língua, bucinador, masseter e temporal. De acordo com a literatura ${ }^{19}$ exercícios isométricos são indicados para fortalecimento dos lábios, bochechas e para uma melhor tensão da língua. $O$ trabaIho mioterápico na SAHOS também deve abranger a musculatura extrínseca e intrínseca da língua, além de fortalecer os músculos do palato mole ${ }^{14}$. A paciente foi orientada sobre a mastigação bilateral alternada, visto que os músculos já se encontravam com força adequada, e não havia qualquer impedi- 
mento para tal. Realizou-se treino funcional com alimento, assim como relatado na literatura, buscando uma harmonia muscular já que os músculos orofaciais possuem uma interrelação e interdependência funcional ${ }^{14}$.

Durante todo o tratamento a paciente realizou com grande empenho todos os exercícios propostos e seguiu cuidadosamente as orientações recebidas.

A literatura caracteriza a SAHOS acima de 5 eventos de apnéia por hora, valor em adultos 1. No exame de polissonografia realizado após o término do tratamento fonoaudiológico, verificou-se que o índice de apnéia e hipopnéia passou de 44 para 3 eventos por hora, não mais caracterizando a SAHOS. Cabe ressaltar que, em média, $75 \%$ dos pacientes que realizam polissonografia têm diagnóstico de SAHOS ${ }^{11}$, indicando que a intervenção fonoaudiológica foi eficaz neste caso. A polissonografia é considerada um exame fundamental para controle da SAHOS, determinando a eficácia das intervenções realizadas ${ }^{10}$.

Em estudos realizados em pacientes com SAHOS percebeu-se que os mesmos apresentam saturação de oxigênio abaixo de $90 \%{ }^{20}$. Os dados da primeira polissonografia demonstram que a saturação média de oxigênio foi de $93 \%$, chegando a uma mínima de $83 \%$, já na segunda polissonografia a saturação média foi de $99 \%$, chegando à mínima de $92 \%$.

Em estudo fonoaudiológico realizado anteriormente notou-se melhora de 40 a $50 \%$ no número de paradas respiratórias, diminuição considerável do ronco e sonolência diurna ${ }^{14}$. No presente estudo notou-se melhora de $93 \%$ no número de paradas respiratórias, bem como diminuição do ronco e ausência de sonolência diurna.

$\mathrm{Na}$ primeira avaliação fonoaudiológica observou-se posição elevada do osso hióide com dorso de língua elevado e com tensão aumentada. De acordo com a literatura, geralmente os pacientes com SAHOS de grau grave apresentam o osso hióide mais baixo que aqueles com SAHOS de grau leve a moderado, apesar de todos terem o posicionamento do osso hióide mais inferiorizado ${ }^{21}$. Acredita-se que a posição elevada do osso hióide observada no presente estudo esteja relacionada com a grande tensão encontrada na região cervical.
A paciente em estudo encontrava-se deprimida, desanimada e com medo de vir a óbito devido às paradas respiratórias durante o sono, visto que a SAHOS é um fator de risco independente para a morte ${ }^{8}$. Realizava atividades domésticas diárias com dificuldade, necessitando de pausas entre as mesmas além de sentir grande fadiga ao realizar pequenas caminhadas, tendo que suspendê-las. Nas últimas sessões começou a relatar melhoras gradativas na sonolência e no cansaço diurno, tornando-se menos ansiosa em relação à sua doença e muito mais disposta na realização das atividades domésticas diárias, inclusive voltando a realizar trabalhos artesanais fora de casa. Ao adequar-se a musculatura da orofaringe, diminuise os despertares noturnos e ronco aumentandose a disposição para desenvolver atividades ${ }^{14}$. Ao final do tratamento a paciente relatou melhora em vários aspectos da sua vida, estando seus familiares satisfeitos em vê-la bem disposta e com ânimo para viver. Este dado demonstra que a redução dos sintomas iniciais proporciona qualidade de vida melhor num curto espaço de tempo nos casos de SAHOS ${ }^{14}$.

Diante dos resultados deste caso sugere-se mais estudos do tratamento fonoaudiológico em pacientes com SAHOS, incluindo amostra maior. É importante também que a Fonoaudiologia consiga definir as técnicas mais eficazes para o tratamento destes casos, uma vez que não há na literatura descrição das técnicas empregadas. Apesar disso, a terapia fonoaudiológica miofuncional mostra-se importante no tratamento da síndrome da apnéia e hipopnéia obstrutiva do sono ${ }^{15}$.

\section{CONCLUSÃO}

Com base nas evidências clínicas encontradas e no resultado da polissonografia verificou-se que, com técnicas específicas de fonoterapia, apesar dos fatores agravantes, foi possível diminuir o número de apnéias e hipopnéias durante o sono do caso em estudo, chegando a um nível não mais considerado como síndrome da apnéia e hipopnéia obstrutiva do sono. O sono da paciente tornou-se tranqüilo e reparador, melhorando consideravelmente sua qualidade de vida e de seus familiares. 


\begin{abstract}
Purpose: to check the contribution of speech therapy in the treatment of a patient with obstructive sleep apnea-hypopnea syndrome. Methods: case description of a sixty-year old, female patient with severe obstructive sleep apnea-hypopnea syndrome that underwent speech therapy treatment for improvement of the apnea/ hypopnea condition, snoring, and day fatigue. Clinical evaluation of orofacial myology and a polysomnography were performed before and after speech therapy. Based on the clinical evaluation, a therapeutic plan was elaborated to provide the patient with cervical relaxation and relaxation of the suprahyoid muscles, for improvement in nasal aeration, tongue strength and position, strengthening of the soft palate muscles and their mobility, as well as the mastication muscles. Alternate bilateral mastication training and lowering of the hyoid bone were included. Results: after twelve-forty-minute sessions of speech therapy, a decrease of cervical tension and relaxation of the suprahyoid muscles were observed. The position of the hyoid bone had been adjusted as well as tongue normotension with the dorsal lowered. The soft palate had normal mobility with adequate mastication. The patient reported important improvement in day fatigue. The result of the second polysomnography indicated a decrease from 44 to 3 events per hour of apnea and hypopnea during sleep, lowering the severe level to an index of breathing disturbance which no longer characterized the disease as sleep apnea. Conclusion: speech therapy was effective in the treatment for this case of obstructive sleep apnea-hypopnea syndrome.
\end{abstract}

KEYWORDS: Sleep Apnea Syndromes; Speech Therapy; Polysomnography

\section{REFERÊNCIAS}

1. American Academy of Sleep Medicine. Sleep: related breathing disorders in adults: recommendations for syndrome definitions and measurements techniques in clinical research. Sleep. 1999; 22:667-89.

2. Gregório LC, Zonato Al. Síndrome da apnéia e hipopnéia obstrutiva do sono. In: Fukuda Y. Otorrinolaringologia: guias de medicina ambulatorial e hospitalar. São Paulo: Manole; 2003. p. 193-202.

3. Mujica RCJ, Mayorga AJ, Llontop GA. Síntomas relacionados a trastornos del sueño em supuestos sanos que asiten a um centro de atención primaria de salud. Med Hered. 2005; 16:31-8.

4. Sociedade Brasileira de Rinologia e Cirurgia Plástica Facial. Atualização otorrinolaringológica em cirurgia de ronco e apnéia do sono. São Paulo: Sociedade Brasileira de Otorrinolaringologia; 2002.

5. Castro JR, Montalvo JH. Sintomas relacionados al síndrome apnea-hipopnea obstructiva del sueño e insomnio. Bol Soc Peru Med Interna. 2000; 13:196-200.

6. Cavallari FEM, Leite MGJ, Mestriner PRE, Couto LGF, Fomin DS, Oliveira JAA. Relação entre hipertensão arterial sistêmica e síndrome da apnéia obstrutiva do sono. Rev Bras Otorrinolaringol. 2002; 68:619-22.

7. Peppard PE, Young T, Palta M, Skatrud J. Prospective study of the association between sleep-dis- ordered breathing and hypertension. N Engl J Med. 2000; 342(19):1378-84.

8. Yaggi HK, Concato J, Kernan WN, Lichtman JH, Brass LM, Mohsenin V. Obstructive sleep apnea as a risk factor for stroke and death. $\mathrm{N}$ Engl $\mathrm{J}$ Med. 2005; 353(19):2034-41.

9. Reimão R, Joo SH. Mortalidade da apnéia obstrutiva do sono. Assoc Med Bras. 2000; 46:52-6.

10. Rueda RS. Diagnóstico del syndrome de apnea obstructive del sueño. Acta Otorrinolaringol Cir Cabeza Cuello. 1999; 27:56-60.

11. American Thoracic Society. Indications and standards for use of nasal continuous positive airway pressure (CPAP) in sleep apnea syndromes. Am J Respir Crit Care Med. 1994; 150(6):1738-45.

12. Munõz AMD. Apnéia do sono e ronco. In: Jafek DW, Starck AK. Segredos em otorrinolaringologia. Porto Alegre: Artmed; 1998. p. 185-90.

13. Zonato Al, Gregório LC. Tratamento cirúrgico da síndrome da apnéia e hipopnéia obstrutivas do sono. In: Sociedade Brasileira de Otorrinolaringologia. Tratado de otorrinolaringologia. São Paulo: Roca; 2003. p. 329-48.

14. Guimarães K. Alterações no tecido mole de orofaringe em portadores da apnéia do sono obstrutiva. J Bras Fonoaudiol. 1999; 1:69-75.

15. Burger RCP, Caixeta EC, Di Ninno CQMS. A relação entre apnéia do sono, ronco e respiração oral. Rev CEFAC. 2004; 6(3):266-71.

16. Oliveira MFR, Souza TGG, Campiotto AR. Contribuição da fonoaudiologia no tratamento de indivíduos roncadores: relato de um caso. In: Marchesan 
IQ, Zorzi JL, Gomes ICD. Tópicos em fonoaudiologia 1997/1998. São Paulo: Lovise; 1998. p. 489-501. 17. Sociedade Brasileira do Sono. Sociedade Brasileira de Rinologia. Sociedade Brasileira de Otorrinolaringologia. I Consenso em ronco e apnéia do sono. São Paulo: Sociedade Brasileira do Sono; 2000.

18. Bacha SMC, Ríspoli CFM. Terapia miofuncional com limitação: uma proposta sistematizada. Rev Soc Bras Fonoaudiol. 2001; 6:65-8.

19. Junqueira $P$, Parro FM, Toledo MR, Araújo RLT, Di Francesco R, Rizzo MC. Conduta fonoaudiológica para pacientes com diagnóstico de rinite alérgica: relato de caso. Rev CEFAC. 2005; 7(3):336-9.

20. Skatvedt O, Akre H, Godtlibsen OB. Nocturnal polysomnography with and without continuous pharyngeal and esophageal pressure measurements. Sleep. 1996; 19(6):485-90.

21. Chaves CBJ. Estudo cefalométrico radiográfico das características crânio-faciais em pacientes com síndrome da apnéia do sono obstrutiva [mestrado]. São Paulo (SP): Faculdade de Ciências Biológicas e da Saúde do Instituto Metodista de Ensino Superior da Federação de Escolas Superiores do ABC; 1997.

Endereço para correspondência:

Rua Francisco Fernandes dos Santos, n. 22 / 102

Belo Horizonte - MG

CEP: $30575-290$

Tel: (31) 32342977

E-mail: fonoleticia@yahoo.com.br 